\title{
CAPPABLE CEA SETS AND RAMSEY'S THEOREM
}

\author{
ASHER M. KACH, MANUEL LERMAN, AND REED SOLOMON
}

ABSTRACT. We begin a search for degree-theoretic properties that might be used to separate Ramsey's Theorem for pairs from its stable version in the Reverse Mathematical sense. This paper introduces the notion of c-cappability and shows that this property cannot be used to obtain such a separation when combined with 2-CEA-ness.

\section{INTRODUCTION}

Let $\mathbb{N}$ denote the natural numbers. Fix $X \subset \mathbb{N}$, and let $[X]^{2}=$ $\{Y \subseteq X:|Y|=2\}$. A 2-coloring $C$ of $[\mathbb{N}]^{2}$ is a function from $[\mathbb{N}]^{2}$ into $\{0,1\}$; such a 2 -coloring is said to be stable if for each $x \in \mathbb{N}$ there exists a $y \in \mathbb{N}$ and a $c \in\{0,1\}$ such that $C(x, z)=c$ for all $z>y$. A set $H \subset \mathbb{N}$ is homogeneous for $C$ if $C$ is constant on $[H]^{2}$. Ramsey's Theorem for pairs, $\mathrm{RT}_{2}^{2}$, states that every 2-coloring of $[\mathbb{N}]^{2}$ has an infinite homogeneous set, and Stable Ramsey's Theorem for pairs, $\mathrm{SRT}_{2}^{2}$, is the analogous statement for stable 2-colorings of $[\mathbb{N}]^{2}$. An excellent summary of the reverse mathematical results dealing with Ramsey's Theorem can be found in [7].

This paper was motivated by an attempt to separate $\mathrm{RT}_{2}^{2}$ from $\mathrm{SRT}_{2}^{2}$, in the sense of Reverse Mathematics. One normally tries to achieve such a separation by building an ideal $\mathbf{I}$ of degrees such that every stable 2-coloring of $[\mathbb{N}]^{2}$ with degree in $\mathbf{I}$ has a homogeneous set in $\mathbf{I}$, but there is a (non-stable) 2-coloring of $[\mathbb{N}]^{2}$ with degree in $\mathbf{I}$ that does not have a homogeneous set in $\mathbf{I}$. Frequently, $\mathbf{I}$ is defined within a set of degrees that is closed under relativization such as the low $_{n}$ degrees. (Note that while the $\mathrm{low}_{n}$ degrees are closed downward and under relativization, they are not closed under join and hence do not form an ideal.) For example, separations from $\mathrm{WKL}_{0}$ are often obtained using the fact that there is an ideal $\mathbf{I}$ contained within the low degree such that the $\omega$-model with second order part $\{X \mid \operatorname{deg}(X) \in \mathbf{I}\}$ is a model of $\mathrm{WKL}_{0}$. Such an ideal suffices to show that $\mathrm{WKL}_{0}$ cannot

2000 Mathematics Subject Classification. Primary: 03C57; Secondary: 03D45, $06 \mathrm{~A} 06$. 
prove either $\mathrm{RT}_{2}^{2}$ or $\mathrm{SRT}_{2}^{2}$. (By results of Hirst, this separation can also be obtained by considering levels of induction in non- $\omega$-models.)

It is not clear whether such ideals within the low or low legrees can $_{2}$ be used to separate $\mathrm{SRT}_{2}^{2}$ and $\mathrm{RT}_{2}^{2}$. With respect to the $\mathrm{low}_{2}$ degrees, Cholak, Jockusch and Slaman [3] proved every computable instance of $\mathrm{RT}_{2}^{2}$ (and hence also every computable instance of $\mathrm{SRT}_{2}^{2}$ ) has a low 2 solution. With respect to the low degrees, while every computable instance of $\mathrm{SRT}_{2}^{2}$ must have a $\Delta_{2}^{0}$ solution (unlike computable instances of $\mathrm{RT}_{2}^{2}$ ), Downey, Hirschfeldt, Lempp and Solomon [6] constructed a computable instance of $\mathrm{SRT}_{2}^{2}$ which has no low solution. (Downey and Hirschfeldt announced recently that they can improve the result by replacing low with $l o w_{2}$.)

The aim of this paper is to begin a search for a smallness property of degrees which can be combined with low $_{2}$-ness to build a separating ideal. By smallness, we mean a property that is low for Reverse Mathematics; a degree is of this type if no degree $\geq \mathbf{0}^{\prime}$ has that property. The property of degrees we consider is c-cappability; a degree $\mathbf{b}$ is said to be $c$-cappable if $\mathbf{b} \neq \mathbf{0}$ and there is a non-zero c.e. degree $\mathbf{a}$ and a CEA degree $\mathbf{d}$ such that $\mathbf{b} \leq \mathbf{d}$ and $\mathbf{a}$ and $\mathbf{d}$ form a minimal pair, i.e., the only degree below both $\mathbf{a}$ and $\mathbf{d}$ is $\mathbf{0}$. (See [8] for the definition of the REA sets and degrees, which have been renamed CEA.). We will show that c-cappability cannot be used to achieve such a separation if we require that the separating degree also be 2-CEA.

When considering lowness properties in the context of Reverse Mathematics, we mean a property not possessed by the degree $\mathbf{0}^{\prime}$. It would be even more desirable if $\mathbf{0}^{\prime}$ is not in the set generated by closing under finitely many applications of relativization. The $\operatorname{low}_{n}$ degrees for each $n$ are examples of sets of degrees with such properties. In searching for other possible properties to consider, we were led, by analogy, to look at the c-cappable degrees. Ambos-Spies, Jockusch, Shore and Soare [1] showed that the c-cappable c.e. degrees (i.e., the cappable c.e. degrees) form a proper ideal in the c.e. degrees, so are low in the sense just mentioned. As the CEA degrees are the closest analog found within the arithmetical degrees to the way the c.e. degrees sit below $\mathbf{0}^{\prime}$, we hoped that the analogy would extend to show that the c-cappable degrees form an ideal within the arithmetical degrees which does not contain $\mathbf{0}^{\prime}$. (Note that $\mathbf{0}^{\prime}$ is not c-cappable.) Futhermore, we hoped that the same would be true under finitely many applications of relativization of c-cappability. The latter hope is refuted by a result of Ambos-Spies, Lempp and Lerman [2] which states that a finite lattice can be embedded into the c.e. degrees preserving least element 0 and greatest element 1 if and only if it can be partitioned into two parts; the first 


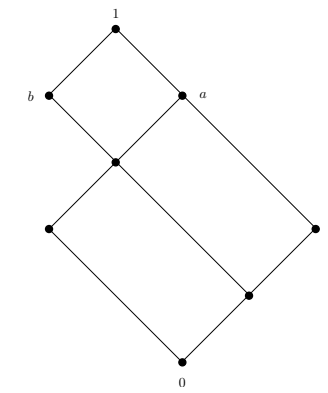

FiguRE 1. Lattice Example

downward closed, containing the ideal generated by the cappable elements of the lattice, and having a greatest element $a$ which is different from the greatest element of the lattice; and the second upward closed and containing a smallest element $b$. Figure 1 exhibits a finite lattice with this property in which $a \vee b=1$, and so shows that $\mathbf{0}^{\prime}$ lies in the ideal generated by the elements that are c-cappable relative to a c-cappable degree.

We next note that Ding and Qian [4] showed that there is a non-zero c.e. degree $\mathbf{a}$ and a non-zero d-c.e. degree $\mathbf{b}$ whose meet is $\mathbf{0}$ and whose join is $\mathbf{0}^{\prime}$. As Lachlan has shown that every d-c.e. degree is 2-CEA, it follows that the ideal generated by the c-cappable degrees contains $\mathbf{0}^{\prime}$.

The failure of the closure under relativization of c-cappability and the fact that $\mathbf{0}^{\prime}$ lies in the ideal generated by the c-cappable degrees still does not preclude the possibility of building an ideal of c-cappable degrees avoiding $\mathbf{0}^{\prime}$; for example, such ideals exist within the $\operatorname{low}_{2}$ degrees. Thus it still makes sense to ask whether every computable stable coloring of pairs has a homogeneous set of c-cappable degree. Our Theorem 10 shows that this, unfortunately, is not the case if we also require that the degree of the separating set be computable from a 2-CEA degree.

Our notation essentially follows that of [9]. We define the use of a computation to be the largest number whose membership in or out of the oracle is used in the computation, and let $B \uparrow u$ denote the string $\sigma \in\{0,1\}^{<\omega}$ of length $u+1$ such that $\sigma \subset B$. (Inclusion for strings is interpreted as extension, and sets are identified with their characteristic functions.)

\section{2. $S R T_{2}^{2}$ AND C-CAPPABILITY}

When we embarked on the research presented in this paper, we had hoped to show at least that every stable 2-coloring of pairs had a ccappable 2-CEA homogeneous set. The main theorem of this section 
shows that this is not the case. Our proof relies on properties of enumerations of $n$-CEA sets, in particular, the case for $n=2$. Our analysis is a generalization of what happens in the c.e. case, so we begin by reviewing it.

To simplify our definitions in this section, it is convenient to assume that all the enumerated sets are infinite. Therefore, rather than enumerating the standard sets $W_{e}$ (possibly relative to some oracle), we enumerate the sets $W_{e} \oplus \mathbb{N}$. We modify the usual enumeration procedure for $W_{e}$ by specifying that for all $s, 2 s+1$ enters $W_{e} \oplus \mathbb{N}$ at stage $s+1$ (with no oracle information in the cases when we are enumerating relative to an oracle). Thus we obtain the property that at every stage at least one number (and possibly more) is enumerated without any nonuniform worries about finite sets. Because we are only concerned with degree properties, this convention is permissible. We also retain the standard property that no numbers are enumerated at stage 0 .

Throughout this section, we use $\subseteq$ to denote the subset relation between sets and $\preceq$ to denote the initial segment relation between strings. When appropriate, we view a set as an infinite string given by its characteristic function. The subscript 1 in the following definitions indicates that we are dealing with 1-CEA (that is, c.e.) sets. The subscript plays no role at this point, but will be relevant later when we extend up the $n$-CEA hierarchy.

Definition 1. Fix a c.e. set $B_{1}$ with an enumeration $\left\{B_{1}^{s}: s \in \mathbb{N}\right\}$, subject to the conventions above. (The definitions to follow depend on this enumeration, but we suppress this dependence in the notation, considering the enumeration fixed.) A stage $t$ is a 1-nondeficiency stage for $B_{1}$ if either $t=0$ or $\min \left\{B_{1}^{t}-B_{1}^{t-1}\right\}<\min \left\{B_{1}-B_{1}^{t}\right\}$. Similarly, for a stage $s$, we say that $t<s$ is a $\langle 1, s\rangle$-nondeficiency stage for $B_{1}$ if either $t=0$ or $\min \left\{B_{1}^{t}-B_{1}^{t-1}\right\}<\min \left\{B_{1}^{s}-B_{1}^{t}\right\}$.

We use the nondeficiency stages to modify the approximation to $B_{1}$ as follows.

Definition 2. The 1-nondeficiency approximation $\left\{\beta_{1}^{s}: s \in \mathbb{N}\right\}$ to $B_{1}$ is defined as follows. For $s=0$, set $\beta_{1}^{0}=\emptyset$. For $s>0$, let $\beta_{1}^{s}$ be such that $\beta_{1}^{s} \preceq B_{1}^{s}$ with length $\min \left\{B_{1}^{s}-B_{1}^{s-1}\right\}$. $\beta_{1}^{s}$ is called the 1-nondeficiency oracle for $B_{1}$ at stage $s$. We say that $t$ is 1 -correct if $\beta_{1}^{t} \preceq B_{1}$ and that $t$ is $\langle 1, s\rangle$-correct if $t<s$ and $\beta_{1}^{t} \preceq \beta_{1}^{s}$.

Notice that the nondeficiency approximation is really a $\Delta_{2}^{0}$ approximation in the sense that when the length of $\beta_{1}^{s}$ shrinks, the approximation temporarily removes numbers which are elements of $B_{1}$. The 
following facts are easy consequences of these definitions for any c.e. set $B_{1}$ with approximations as above.

Lemma 1. If $0=s_{0}<s_{1}<\cdots$ are the 1-nondeficiency stages for $B_{1}$, then $\beta_{1}^{s_{0}} \preceq \beta_{1}^{s_{1}} \preceq \cdots$. Furthermore, for any fixed $s$, if $0=s_{0}<$ $s_{1}<\cdots s_{k}$ are the $\langle 1, s\rangle$-nondeficiency stages for $B_{1}$ then $s_{k}<s$ and $\beta_{1}^{s_{0}} \preceq \beta_{1}^{s_{1}} \preceq \cdots \preceq \beta_{1}^{s_{k}} \preceq \beta_{1}^{s}$.

Lemma 2. A stage $t$ is a 1-nondeficiency stage for $B_{1}$ if and only if $t$ is 1-correct. For $t<s, t$ is a $\langle 1, s\rangle$-nondeficiency stage for $B_{1}$ if and only if $t$ is $\langle 1, s\rangle$-correct.

Lemma 3. For any $t<s$, if $t$ is not $\langle 1, s\rangle$-correct, then $t$ is not $\langle 1, r\rangle$ correct for any $r \geq s$ and $t$ is not 1-correct.

We generalize this concept to 2-CEA sets and then through the CEA hierarchy. We separate the 2-CEA case because it gives the general picture and we will need a special property of the 2-CEA level of the hierarchy for the main theorem of this section.

Consider a set $B_{2}$ which is c.e. relative to a c.e. set $B_{1}$. By convention, we assume that $B_{2}$ has the form $B_{2}=W_{e}^{B_{1}} \oplus \mathbb{N}$ for some $e$ and that the enumeration of $B_{2}$ from $B_{1}$ has the property that $2 s+1$ is enumerated into $B_{2}$ at stage $s+1$ using no oracle information. We fix a computable approximation $B_{2}^{s}$ to $B_{2}$ by $B_{2}^{0}=\emptyset$ and for $s>0, B_{2}^{s}$ is the set of numbers enumerated using the 1-nondeficiency oracle $\beta_{1}^{s}$. Notice that this approximation has the property that $B_{2}^{s+1}-B_{2}^{s} \neq \emptyset$ for all $s$.

Definition 3. Let $B_{2}$ be c.e. relative to a c.e. set $B_{1}$ with a computable approximation $B_{2}^{s}$ as described above. Let $0=s_{0}<s_{1}<\cdots$ be the sequence of 1-nondeficiency stages for $B_{1} . t$ is a 2-nondeficiency stage for $B_{2}$ if $t=0$ or $t=s_{i}$ for some $i>0$ and $\min \left\{B_{2}^{s_{i}}-B_{2}^{s_{i-1}}\right\}<\min \left\{B_{2}-\right.$ $\left.B_{2}^{s_{i}}\right\}$. For $t<s$, let $0=s_{0}<s_{1}<\cdots s_{k}$ the $\langle 1, s\rangle$-nondeficiency stages and note that $s_{k}<s$. $t$ is a $\langle 2, s\rangle$-nondeficiency stage for $B_{2}$ if $t=0$ or $t=s_{i}$ for some $0<i \leq k$ and $\min \left\{B_{2}^{s_{i}}-B_{2}^{s_{i-1}}\right\}<\min \left\{B_{2}^{s}-B_{2}^{s_{i}}\right\}$.

By Lemma 1, if $s_{0}<s_{1}<\cdots$ are the 1-nondeficiency stages for $B_{1}$, then $B_{2}^{s_{0}} \subseteq B_{2}^{s_{1}} \subseteq \cdots$. Furthermore if $s$ is fixed and $s_{0}<s_{1}<\cdots<s_{k}$ are the $\langle 1, s\rangle$-nondeficiency stages for $B_{1}$, then $B_{2}^{s_{0}} \subseteq B_{2}^{s_{1}} \subseteq \cdots \subseteq$ $B_{2}^{s_{k}} \subseteq B_{2}^{s}$. We can now define the 2-nondeficiency approximation to $B_{2}$.

Definition 4 . Let $B_{2}$ be c.e. relative to a c.e. set $B_{1}$ with the approximations as above. The 2-nondeficiency approximation $\left\{\beta_{2}^{s} \mid s \in \mathbb{N}\right\}$ to $B_{2}$ is defined as follows. For $s=0, \beta_{2}^{0}=\emptyset$. For $s>0$, let $t_{2}^{s}<s$ denote the largest $\langle 2, s\rangle$-nondeficiency stage. (By the comments above, $B_{2}^{t_{2}^{s}} \subseteq B_{2}^{s}$.) Let $\beta_{2}^{s} \preceq B_{2}^{s}$ with length $\min \left\{B_{2}^{s}-B_{2}^{t_{2}^{s}}\right\}$. 
Since our real goal is to deal with 2 -CEA sets, let $B_{1}$ be a c.e. set and let $B_{2}$ be c.e. relative to $B_{1}$ with the fixed approximations and terminology as above. Consider the 2-CEA set $B=B_{1} \oplus B_{2}$. Abusing terminology slightly, we define the 2-nondeficiency approximation to $B$ as $\left\{\beta^{s} \mid s \in \mathbb{N}\right\}$ by $\beta^{0}=\emptyset$ and $\beta^{s}=\beta_{1}^{s} \oplus \beta_{2}^{s}$ for $s>0$. (Since $\beta_{1}^{s}$ and $\beta_{2}^{s}$ need not have the same length, we introduce a formal symbol $\lambda$ with the intention that $\beta_{i}^{s}(k)=\lambda$ indicates that $\beta_{i}^{s}$ has no commitment concerning whether $k$ is in or out of the approximated set. We extend the shorter string using $\lambda$ symbols to make them have the same length. When comparing strings $\alpha$ and $\delta$ which may contain $\lambda$, we write $\alpha \preceq \delta$ if for all $k<|\alpha|$, if $\alpha(k) \neq \lambda$, then $k<|\delta|$ and $\alpha(k)=\delta(k)$.)

Definition 5. Let $B=B_{1} \oplus B_{2}$ be as above. $t$ is a 2-nondeficiency stage for $B$ if $t$ is a 2-nondeficiency stage for $B_{2}$ (and hence is also a 1nondeficiency stage for $\left.B_{1}\right)$. For a fixed $s, t<s$ is a $\langle 2, s\rangle$-nondeficiency stage for $B$ if $t$ is a $\langle 2, s\rangle$-nondeficiency stage for $B_{2}$ (and hence is also a $\langle 1, s\rangle$-nondeficiency stage for $\left.B_{1}\right)$. $t$ is 2-correct if $\beta^{t} \preceq B$. For any fixed $s, t<s$ is $\langle 2, s\rangle$-correct if $\beta^{t} \preceq \beta^{s}$.

From these definitions, it is easy to verify the analogs of Lemmas 1 and 2 for a 2 -CEA set $B=B_{1} \oplus B_{2}$ as above.

Lemma 4. If $0=s_{0}<s_{1}<\cdots$ are the 2-nondeficiency stages for $B_{1} \oplus B_{2}$, then $\beta^{s_{0}} \preceq \beta^{s_{1}} \preceq \cdots$. Furthermore, for any fixed $s$, if $0=s_{0}<s_{1}<\cdots s_{k}$ are the $\langle 2, s\rangle$-nondeficiency stages for $B_{1} \oplus B_{2}$ then $s_{k}<s$ and $\beta^{s_{0}} \preceq \beta^{s_{1}} \preceq \cdots \preceq \beta^{s_{k}} \preceq \beta^{s}$.

Lemma 5. A stage $t$ is a 2-nondeficiency stage for $B_{1} \oplus B_{2}$ if and only if $t$ is 2-correct. For $t<s, t$ is a $\langle 2, s\rangle$-nondeficiency stage for $B_{1} \oplus B_{2}$ if and only if $t$ is $\langle 2, s\rangle$-correct.

The analog of Lemma 3 fails in the case of 2 -CEA sets $B_{1} \oplus B_{2}$. It is easy to verify that if $s$ is 2-correct, then there are infinitely many stages $t>s$ at which $s$ is $\langle 2, t\rangle$-correct, but it is not true is general that if $s$ is 2-correct, then $s$ is $\langle 2, t\rangle$-correct at all stages $t>s$. However, we can get some additional correctness information which will be crucial to our proof.

Lemma 6. If $r$ is not 2-correct, then there are only finitely many stages $s>r$ such that $r$ is $\langle 2, s\rangle$-correct.

Proof. If $r$ fails to be 2 -correct because $\beta_{1}^{r} \npreceq B_{1}$, then the conclusion follows immediately. Assume that $\beta_{1}^{r} \preceq B_{1}$ and therefore $B_{2}^{r} \subseteq B_{2}$. We must have $\beta_{2}^{r} \npreceq B_{2}$ so there is a number $n<\left|\beta_{2}^{r}\right|$ such that $n \in B_{2}$ and $\beta_{2}^{r}(n)=0$. Fix the least such $n$ and fix a stage $t>r$ such that 
$\beta_{1}^{t} \preceq B_{1}$ and $\beta_{1}^{t}$ contains the use for the enumeration of $n$ into $B_{2}$. For all $s \geq t, \beta^{r} \npreceq \beta^{s}$ and hence the conclusion follows.

Lemma 7. Let $r^{\prime}<r<s$.

(1) If $r^{\prime}$ is $\langle 2, r\rangle$-correct and $r$ is $\langle 2, s\rangle$-correct, then $r^{\prime}$ is $\langle 2, s\rangle$ correct.

(2) If $r$ is $\langle 2, s\rangle$-correct and $r^{\prime}$ is not $\langle 2, s\rangle$-correct, then $r^{\prime}$ is not $\langle 2, r\rangle$-correct.

Proof. To prove the first statement, notice that by assumption, $\beta^{r^{\prime}} \preceq$ $\beta^{r}$ and $\beta^{r} \preceq \beta^{s}$. Therefore, $\beta^{r^{\prime}} \preceq \beta^{s}$ as required. The second statement follows immediately from the first statement.

Lemma 8. Let $r^{\prime}<r<s$. If $r^{\prime}$ is $\langle 2, s\rangle$-correct but not $\langle 2, r\rangle$-correct, then $r$ is not $\langle 2, t\rangle$-correct for any $t \geq s$ (and hence is not 2-correct).

Proof. First, consider the situation at the $B_{1}$ level. Since $r^{\prime}$ is $\langle 2, s\rangle$ correct, and hence $\langle 1, s\rangle$-correct, $\beta_{1}^{r^{\prime}} \preceq \beta_{1}^{s}$. Hence by Lemma $3, \beta_{1}^{r^{\prime}} \preceq$ $\beta_{1}^{r}$. Therefore, in order to have $\beta^{r^{\prime}} \npreceq \beta^{r}$, we must have $\beta_{2}^{r^{\prime}} \npreceq \beta_{2}^{r}$.

Since $\beta_{1}^{r^{\prime}} \preceq \beta_{1}^{r}$, it follows that $B_{2}^{r^{\prime}} \subseteq B_{2}^{r}$. Therefore, to have $\beta_{2}^{r^{\prime}} \npreceq \beta_{2}^{r}$, we must have a number $n<\left|\beta_{2}^{r^{\prime}}\right|$ enter $B_{2}^{r}$ with oracle $\beta_{1}^{r}$. However, since $\beta_{2}^{r^{\prime}} \preceq \beta_{2}^{s}$, we must have $n \notin B_{2}^{s}$. In order for $n$ to leave the approximation to $B_{2}$ between stages $r$ and $s$, a number $<\left|\beta_{1}^{r}\right|$ must enter $B_{1}$ between stages $r$ and $s$. Therefore, $\beta_{1}^{r} \npreceq \beta_{1}^{s}$ and hence by Lemma $3, \beta^{r}$ cannot be even $\langle 1, t\rangle$-correct for any $t \geq s$.

This method of approximation using nondeficiency oracles can be continued by induction through the $n$-CEA hierarchy. Given the nondeficiency approximation $\left\{\beta^{s} \mid s \in \mathbb{N}\right\}$ for an $n$-CEA set $B=B_{1} \oplus \cdots \oplus B_{n}$ and a set $B_{n+1}$ which is c.e. in $B$ (and of the form $W_{e}^{B} \oplus \mathbb{N}$ by convention), we fix an enumeration of $B_{n+1}$ such that $B_{n+1}^{s}$ consists of the elements enumerated with oracle $\beta^{s}$ (and the convention than $2 s+1$ enters $B_{n+1}$ at stage $s+1$ with no oracle information). We define the $n+1$-nondeficiency stages by thinning the $n$-nondeficiency stages as above and we define the $\langle n+1, s\rangle$-nondeficiency stages by thinning the $\langle n, s\rangle$-nondeficiency stages as above. The $n+1$-nondeficiency approximation $\left\{\beta_{n+1}^{s} \mid s \in \mathbb{N}\right\}$ is defined (for $s>0$ ) by letting $t_{n+1}^{s}$ be the largest $\langle n+1, s\rangle$-nondeficiency stage and setting $\beta_{n+1}^{s} \preceq B_{n+1}^{s}$ with length $\min \left\{B_{n+1}^{s}-B_{n+1}^{t_{n+1}^{s}}\right\}+1$. The analogs of Lemmas 4 and 5 hold by induction for all $n$, but the analogs of Lemmas 6 and 8 fail for $n \geq 3$.

We also note the following. 
Remark 9. It is easy to show and well-known that finding an infinite homogeneous set for a computable stable coloring of pairs that is computable in a given degree is equivalent to finding an infinite subset of a given $\Delta_{2}^{0}$ set or its complement computable in that degree.

Theorem 10. There is a computable stable coloring of pairs that does not have a homogeneous set computable from a c-cappable 2-CEA set.

Proof. By Remark 9, it suffices to show that there is a $\Delta_{2}^{0}$ set $D$ such that neither $D$ nor its complement, $\bar{D}$, has a subset computable from a c-cappable 2-CEA degree. Let $\left\{\varphi_{e}: e \in \mathbb{N}\right\}$ be an effective enumeration of all computable partial functions. It suffices to satisfy the following requirements for all c.e. sets $A, 2$-CEA sets $B=B_{1} \oplus B_{2}$ and computable partial functionals $\Gamma$ (A and $\mathrm{B}$ come with fixed enumerations $\left\{A^{s}: s \in \mathbb{N}\right\}$ and $\left\{\beta_{i}^{s}: i \in\{1,2\} \& s \in \mathbb{N}\right\}$, respectively):

$$
\begin{array}{r}
R_{A, B, \Gamma, D}:(|\Gamma(B)|=\infty \& \Gamma(B) \subset D \& A \text { non-computable }) \Rightarrow \\
\exists C\left(C \leq_{T} A \& C \leq_{T} B \& \forall e\left(C \neq \varphi_{e}\right)\right) ; \\
R_{A, B, \Gamma, \bar{D}}:(|\Gamma(B)|=\infty \& \Gamma(B) \subset \bar{D} \& A \text { non-computable }) \Rightarrow \\
\exists C\left(C \leq_{T} A \& C \leq_{T} B \& \forall e\left(C \neq \varphi_{e}\right)\right) .
\end{array}
$$

Let $C_{A, B, \Gamma, D}$ and $C_{A, B, \Gamma, \bar{D}}$ be the sets constructed to satisfy these requirements. We subdivide each requirement into infinitely many subrequirements, one for each $e$ :

$$
\begin{array}{r}
R_{A, B, \Gamma, D, e}:(|\Gamma(B)|=\infty \& \Gamma(B) \subset D \& A \text { non-computable }) \Rightarrow \\
\left.C_{A, B, \Gamma, D} \leq_{T} A \& C_{A, B, \Gamma, D} \leq_{T} B \& C_{A, B, \Gamma, D} \neq \varphi_{e}\right) ; \\
R_{A, B, \Gamma, \bar{D}, e}:(|\Gamma(B)|=\infty \& \Gamma(B) \subset \bar{D} \& A \text { non-computable }) \Rightarrow \\
\left.C_{A, B, \Gamma, \bar{D}} \leq_{T} A \& C_{A, B, \Gamma, \bar{D}} \leq_{T} B \& C_{A, B, \Gamma, \bar{D}} \neq \varphi_{e}\right) .
\end{array}
$$

We fix an effective ordering $\left\{R_{i}: i \in \mathbb{N}\right\}$ of all subrequirements, and refer to these as requirements. We say that $R_{i}$ has higher priority than $R_{j}$ if $i<j$. We also fix a computable partition $\left\{S_{i}: i \in \mathbb{N}\right\}$ of $\mathbb{N}$ in which each $S_{i}$ is infinite.

Requirements will be in one of two states; a waiting state or a diagonalization state. At each stage $s$ of the construction, we will have a subset $P_{i}^{s}$ of $\{j: j \leq s\}$, and requirements may have designated oracles and designated disruptors. Action taken to satisfy requirements will be defined in terms of configurations and permission. Fix a requirement $R_{i}=R_{A, B, \Gamma, D, e}$. (Everything done henceforth for this requirement applies to requirements $R_{A, B, \Gamma, \bar{D}, e}$, and is obtained by interchanging $D$ 
and $\bar{D}$.) For fixed $i$, we say that $t$ is $i$-configured at stage $s$ if $t<s$ and $\left|\Gamma^{t}\left(\beta^{t}\right) \cap[i, \infty)\right| \geq i+1$. We say that $x \in S_{i}$ is $A$-permitted for $i$ at stage $s$ if $\varphi_{e}^{s}(x) \downarrow=0$, and $\min \left\{A^{s}-A^{s-1}\right\} \leq x$. Note that if $t$ is $i$-configured at stage $r<s$ then $t$ is $i$-configured at stage $s$.

The idea for satisfying $R_{i}$ is as follows. At the first stage $s$ at which some $x \in S_{i}$ is $A$-permitted for $i$, we place $x \in C^{s+1}=C_{A, B, \Gamma, D}^{s+1}$. If no such $s$ exists, then either $A$ is computable or $\left\{x \in S_{i}: \varphi_{e}(x) \downarrow=0\right\}$ is finite; in the latter case, either $\varphi_{e}$ is not total, or we will have an $x \in S_{i}$ such that $\varphi_{e}(x) \downarrow \neq 0=C(x)$. So suppose that $x$ exists. $C \leq_{T} A$ by a standard permitting argument. The argument that $C \leq_{T} B$ is a bit more complex, and assumes that $\Gamma(B)$ is an infinite subset of $D$. We first argue that there can be no $t<s$ such that $\beta^{t}$ is correct and $t$ is $i$-configured at stage $s$, else we would later place the designated disruptor for $\beta^{t}$ into $\bar{D}$, thereby forcing $\Gamma(B) \nsubseteq D$. But if $\Gamma(B)$ is an infinite subset of $D$, then there must be a stage $t$ at which $\beta^{t}$ is correct and $t$ is $i$-configured at stage $t$. By the previous sentence, we must have $t \geq s$. But $t$ can be found computably from $B$, so any number in $S_{i}$ that is placed into $C$ through action for $R_{i}$ must be in $C^{t}$. This provides a computation of $C$ from a $B$ oracle.

\section{The Construction}

Every parameter defined for a requirement at stage $s-1$ has the same value at stage $s$ unless the parameter is redefined during stage $s-1$.

Stage 0: For all $i, R_{i}$ is in the waiting state, $D^{0}=\emptyset$, and $P_{i}^{0}=\emptyset$.

Stage $s>0$ : We proceed by a subinduction on $\{i: i \leq s\}$. Fix $i$. We follow the first case below which applies.

Case 1: $R_{i}$ is in the waiting state and some $x \in S_{i}$ is $A$-permitted at stage $s$. Fix the smallest such $x$, and place $x \in C_{A, B, \Gamma, D}^{s+1}$. If $s$ is $i$-configured, set $P_{i}^{s+1}=P_{i}^{s} \cup\{s\} . R_{i}$ is placed in the diagonalization state at all stages $>s$.

Case 2: $R_{i}$ is in the diagonalization state and has some $y$ as its designated disruptor at stage $s-1$, and there is a $j<i$ that has $y$ as its designated disruptor at stage $s$. Fix $t$ such that $\beta^{t}$ is the designated oracle for $R_{i}$ at stage $s$. Fix the smallest $z \in \Gamma^{t}\left(\beta^{t}\right) \cap[i, \infty)$ that is not a designated disruptor for any $k<i$ at stage $s$; $z$ becomes the designated disruptor for $R_{i}$ at stage $s$. (We will show later that such a $z$ exists.) We specify that $z \in D^{s+1}$ if and only if $y \in D^{s}$. $R_{i}$ remains in the diagonalization state at stage $s$ with the same designated oracle. 
We now follow Case 3 if the conditions for following that case apply, and otherwise complete step $i$ of the subinduction.

Case 3: $R_{i}$ is in the diagonalization state at stage $s-1$ and there is an $r \in P_{i}^{s}$ such that $\beta^{r}$ is $\langle 2, s\rangle$-correct. $\beta^{r}$ becomes the designated oracle for $R_{i}$ for the smallest such $r$, and we set $P_{i}^{s+1}=P_{i}^{s} \cap[0, r)$. Fix the smallest $z \in \Gamma^{r}\left(\beta^{r}\right) \cap[i, \infty)$ that is not a designated disruptor for any $k<i$ at stage $s$; $z$ becomes the designated disruptor for $R_{i}$ at stage $s$. (We will show later that such a $z$ exists.) Place $z \in \bar{D}^{s+1}$ if $R_{i}$ is a requirement of the form $R_{A, B, \Lambda, D, e}$, and place $z \in D^{s+1}$ if $R_{i}$ is a requirement of the form $R_{A, B, \Lambda, \bar{D}, e} \cdot R_{i}$ remains in the diagonalization state at stage $s$.

Case 4: Otherwise. If $R_{i}$ is in the waiting state at the end of stage $s-1$ and $s$ is $i$-configured, then we set $P_{i}^{s+1}=P_{i}^{s} \cup\{s\}$. In all cases, $R_{i}$ retains the state it had at stage $s-1$, and nothing else is changed.

This completes the construction. Note that each requirement has at most one designated disruptor at a given stage, and that if $t$ is $i$-configured, then $\left|\Gamma^{t}\left(\beta^{t}\right) \cap[i, \infty)\right| \geq i+1$. Hence the construction can always find a new designated disruptor when it needs to do so. Furthermore, only $R_{j}$ for $j<i$ can cause designated disruptors for configurations for $R_{i}$ to change, and any such $R_{j}$ can do so at only finitely many stages. Thus each configuration for $R_{i}$ has a final designated disruptor. These final designated disruptors will not be moved from $D$ to $\bar{D}$ until some $x$ is $A$-permitted for $i$, and if such a permission occurs at stage $s$, then such movement can only be caused by $t<s$, and each such $t$ will cause a single designated disruptor to move, and will do so at most once. Hence $D$ is $\Delta_{2}^{0}$.

We note that Case 1 is followed for a given requirement $R_{i}$ at most once, and that once it is followed at stage $s$, the sequence $\left\langle P_{i}^{t}: t \geq s\right\rangle$ is non-increasing, so has a limit. Thus Case 3 can be followed only finitely often for $R_{i}$. Case 2 is followed for $R_{i}$ only when Case 3 is followed for $R_{j}$ for some $j<i$. It follows by induction that Case 4 will be followed for $R_{i}$ at all but finitely many stages.

We now show that $R_{i}$ is satisfied for all $i$, and that this satisfaction is uniform for all subrequirements of the same master requirement $R$. Again, we only consider the case wherein $R_{i}=R_{A, B, \Gamma, D, e}$; a symmetric proof for the case in which $R_{i}=R_{A, B, \Gamma, \bar{D}, e}$ is obtained by interchanging $D$ and $\bar{D}$. Fix $i$. We may assume that $|\Gamma(B)|=\infty, \Gamma(B) \subseteq D$ and $A$ is not computable, else the satisfaction of $R_{i}$ is immediate. Under this assumption, we show that $C_{A, B, \Gamma, D} \leq_{T} A, B$ and $C_{A, B, \Gamma, D} \neq \varphi_{m}$ for all $m$. 
We begin by showing that $C=C_{A, B, \Gamma, D} \neq \varphi_{e}$; we may assume that $\varphi_{e}$ is total, else there is nothing to show. We also fix a stage $s$ such that all $R_{j}$ for $j<i$ follow Case 4 of the construction at all stages $t \geq s$. It follows that each configuration $\beta^{r}$ for such an $R_{j}$, has a designated disruptor at stage $t \geq \max \{r, s\}$ if and only if it has one at stage $\max \{r, s\}$, and that any designated disruptor at stage $\max \{r, s\}$ remains the designated disruptor at all later stages. As $|\Gamma(B)|=\infty$, it follows from Lemma 5 that there are infinitely many 2-correct stages $t$, and at all but finitely many of these stages, $\left|\Gamma^{t}\left(\beta^{t}\right)\right| \cap[i, \infty) \geq i+1$.

First assume that $R_{i}$ is in the waiting state at all stages. As $S_{i} \cap S_{j}=$ $\emptyset$ if $i \neq j$, we see that no number in $S_{i}$ is placed in $C$ at any stage. Thus if there is an $x \in S_{i}$ such that $\varphi_{e}(x) \neq 0$, then we will have an $x$ such that $\varphi_{e}(x) \neq 0=C(x)$, so $\varphi_{e} \neq C$. Otherwise, there are arbitrarily large $x \in S_{i}$ such that $\varphi_{e}(x)=0$, so as $A$ is not computable, there would be a stage $t$ at which some $x$ is $A$-permitted for $i$, so we would place $R_{i}$ in the diagonalization stage at stage $t$, contrary to our assumption. Thus this case cannot occur.

Now assume that $R_{i}$ is in the diagonalization state at some stage $t$. Then some $x$ such that $\varphi^{s}(x)=0$ is placed in $C^{s+1}$, so again we see that $\varphi_{e} \neq C$.

A standard permitting argument shows that for all $x$, for the least stage $s$ such that $A^{s} \uparrow x=A \uparrow x, x \in C^{s+1}$ if and only if $x \in C$. Thus $C \leq_{T} A$.

We now describe how to compute $C$ using a $B$ oracle. Uniformly in $x$, we can effectively find the unique $j$ such that $x \in S_{j}$. Fix $x$ and the unique $j$ such that $x \in S_{j}$. If $R_{i} \neq R_{A, B, \Gamma, D, m}$ for any $m$, then $x \notin C$. Hence, without loss of generality, we may suppose that $x \in S_{i}$, i.e., that $j=i$. Use a $B$ oracle to find the least 2 -correct stage $t$ such that $\left|\Gamma^{t}\left(\beta^{t}\right)\right| \geq i+1$. By our assumptions and the fact that there are infinitely many 2 -correct stages, such a stage must exist.

We claim that $x \in C$ if and only if $x \in C_{t+1}$. For suppose that $x \in C$. Then $x$ is placed into $C$ at some stage $s$ at which $x$ is $A$-permitted for $i$. If $s \leq t$, then $x \in C_{t+1}$ as required. Suppose $t<s$ and we derive a contradiction. Since $R_{i}$ remains in the waiting state until $x$ is placed into $C$ and since $t$ is $i$-configured, $t \in P_{i}^{s}$. We show that eventually $\beta^{t}$ is chosen as the designated oracle for $R_{i}$. To see why, let $s^{\prime} \geq s$ be the first stage at which $R_{i}$ picks a designated oracle and let $\beta^{r}$ (for some $r \in P_{i}^{s^{\prime}}=P_{i}^{s}$ ) be the designated oracle chosen. (Since $t$ is $\left\langle 2, s^{\prime}\right\rangle$-correct for infinitely many stages $s^{\prime}$ and since $t \in P_{i}^{s}$, there must be such a stage.) 
We claim that $t \leq r$. For a contradiction, suppose that $r<t$. Then $r<t<s^{\prime}$ and $r$ is $\left\langle 2, s^{\prime}\right\rangle$-correct. By Lemma 8, if $r$ is not $\langle 2, t\rangle$ correct, then $t$ is not 2-correct. Therefore, $r$ must be $\langle 2, t\rangle$-correct and hence $r$ is 2-correct since $\beta^{r} \preceq \beta^{t} \preceq B_{1} \oplus B_{2}$. This contradicts the minimality property of $t$.

If $r=t$, then we have shown that $\beta^{t}$ is eventually chosen as the designated oracle for $R_{i}$. If $t<r$, then $r$ cannot be 2-correct. (If $r$ is 2-correct, then $\beta^{t} \preceq \beta^{r} \preceq \beta^{s^{\prime}}$. However, if $t$ is $\left\langle 2, s^{\prime}\right\rangle$-correct, then we would have chosen $\beta^{t}$ as the designated oracle at stage $s^{\prime}$.) Therefore, there must be a later stage $s^{\prime \prime}$ and a index $r^{\prime}<r$ such that $r^{\prime} \in P_{i}^{s^{\prime \prime}}=P_{i}^{s} \cap[0, r)$ such that $r^{\prime}$ is $\left\langle 2, s^{\prime \prime}\right\rangle$-correct. As above, $t \leq r^{\prime}$. Since $r^{\prime}$ was not chosen as stage $s^{\prime}, r^{\prime}$ is not $\left\langle 2, s^{\prime}\right\rangle$-correct and hence by Lemma 7 (since $r^{\prime}<r<s^{\prime}$ ), $r^{\prime}$ is also not $\langle 2, r\rangle$-correct. We can now apply Lemma 8 to the stages $r^{\prime}<r<s^{\prime \prime}$ to conclude that $r$ is not $\langle 2, p\rangle$-correct for any $p \geq s^{\prime \prime}$. (Therefore, it was safe to define $P_{i}^{s^{\prime \prime}}=P_{i}^{s} \cap\left[0, r^{\prime}\right)$.) If $t=r^{\prime}$, then we have shown that $\beta^{t}$ is eventually chosen as the designated oracle. If $t<r^{\prime \prime}$, then we repeat this argument defining successive new designated oracles until $\beta^{t}$ is chosen.

Once $\beta^{t}$ is chosen as the designated oracle, it remains the designated oracle forever (by Lemma 8 and the arguments given in the previous paragraph). We have shown that $\beta^{t}$ has a final designated disruptor $y$ for $i$. By construction, $y \in \bar{D}$ but (since $\beta^{t}$ is 2-correct) $\Gamma(B ; y)=$ $\Gamma^{t}\left(\beta^{t}\right)=1$. Therefore, $\Gamma(B) \nsubseteq D$ contrary to our assumption. We conclude that $x \in C$ if and only if $X \in C^{t+1}$, so $C \leq_{T} B$.

The proof of Theorem 10 does not generalize to the 3-CEA case. The problem for the 3-CEA case lies in the fact that we may have several pairwise incompatible approximations to $B$, each of which looks correct at infinitely many stages; this prevents us from making $D$ a $\Delta_{2}^{0}$ set. We have not yet looked at the non-stable case. Thus we conclude with some questions.

Question 1. For which integers $n$, if any, is it true that every computable stable 2-coloring of $\omega^{2}$ has a c-cappable $n$-CEA homogeneous set?

We have a similar question for the non-stable case.

Question 2. For which integers $n$, if any, is it true that every computable 2-coloring of $\omega^{2}$ has a c-cappable $n$-CEA homogeneous set? 


\section{REFERENCES}

[1] Ambos-Spies, K., Jockusch, C. G. Jr., and Shore, R. A., and Soare, R. I., An algebraic decomposition of the recursively enumerable degrees and the coincidence of several degree classes with the promptly simple degrees, Trans. Amer. Math. Soc. 281 (1984), 109-128.

[2] Ambos-Spies, K., Lempp, S., and Lerman, M., Lattice embeddings into the r.e. degrees preserving 0 and 1, J. London Math. Soc. (2) 49 (1994), 1-15.

[3] Cholak, Peter A., Jockusch, Carl G., and Slaman, Theodore A., On the strength of Ramsey's Theorem for pairs, J. Symbolic Logic 66 (2001), 1-55.

[4] Ding, D. and Qian L., Lattice embedding into d-r.e. degrees preserving 0 and 1,In: Proceedings of the Sixth Asian Logic Conference (Chong, Feng, Ding, Huang and Yasugi eds., World Scientific Press, Singapore, 1998, pp. 67-81.

[5] Downey, R., D-r.e. degrees and the non-diamond theorem, Bull. London Math. Soc. 21 (1989), 43-50.

[6] Downey, R., Hirschfeldt D., Lempp, S. and Solomon, D. R., A $\Delta_{2}^{0}$ set with no infinite low set contained in it or its complement, J. Symbolic Logic 66(3) (2001), 1371-1381.

[7] Hirschfeldt, Denis R., Jockusch, Jr., Carl G., Kjos-Hanssen, Bjørn; Lempp, Steffen, and Slaman, Theodore A., The strength of some combinatorial principles related to Ramsey's Theorem for pairs, In: Computational Prospects of Infinity, Part II: Presented Talks, World Scientific Press, Singapore, 2008, pp. 143-161.

[8] Jockusch, Jr., Carl G., and Shore, Richard A., Pseudo-Jump Operators I: the r.e. case, Trans. Amer. Math. Soc. 275 (1983), 599-610.

[9] Soare, Robert I., Recursively Enumerable Sets and Degrees, Perspectives In Mathematical Logic, Springer-Verlag, Berlin-Heidelberg-New York, 1987.

Department of Mathematics, University of Connecticut, Storrs, CT 06269-3009

E-mail address: kach@math.uconn.edu

Department of Mathematics, University of Connecticut, Storrs, CT 06269-3009

E-mail address: lerman@math.uconn.edu

Department of Mathematics, University of Connecticut, Storrs, CT 06269-3009

E-mail address: solomon@math.uconn.edu 